R.I.M.E.L. In the illustration by Moe and Nellhaus (1970) the ocular movements recorded seem slow rather than rapid and seem to be independent in each eye (an unusual phenomenon in any disease); moreover the E.M.G. shows rhythmic grouping of muscle action potentials.

In any consideration of involuntary movements, whether at limb or eye level, the electrographic features represent a permanent record of the abnormal motor phenomena. In the differential diagnosis of R.I.M.E.L. the E.E.G. and E.O.G. features are essential physical signs, which differ from the findings in myoclonic epilepsy, the cerebellar ataxias, tremors, and the choreiform syndromes even if the aetiological factors remain at present obscure.

We are indebted to our colleagues at the Hospital for Sick Children and in particular to Drs. E. Brett, Macdonald Critchley, the late Paul Sandifer, and J. Wilson. Support from Action for the Crippled Child is gratefully acknowledged.

\section{References}

Baringer, J. R., Sweeney, V. P., and Winkler, G. F. (1968). Brain, 91, 473. Dyken, P., and Kolár, O. (1968). Brain, 91, 305.

Gastaut, in (1968). Revue Neurologique, 119, 1.

Halliday, A. M. (1967). Brain, 90, 241.

Kinsbourne, M. (1962). Fournal of Neurology, Neurosurgery and Psychiatry, 25,271 .

Lemerle, J., Lemerle, M., Aicardi, J., Messica, C., and Schweisguth, O. (1969). Archives Françaises de Pédiatrie, 26, 547

Moe, P. G., and Nellhaus, G. (1970). Neurology, 8, 756.

Pampiglione, G. (1956). Proceedings of the Electrophysiological Technologists Association, $7,20$.

Pampiglione, G. (1964). Archives of Disease in Childhood, 39, 558.

Pampiglione, G. (1966). Fournal of the Neurological Sciences, 3, 525.

Wilson, S. A. K. (1940). Neurology. London, Arnold.

\title{
False-negative Results in Brain Scanning
}

\author{
E. H. BURROWS
}

British Medical fournal, 1972, 1, 473-476

\section{Summary}

There were 118 false-negative results in a series of 847 cases of proved intracranial lesions subjected to brain scanning. In patients with neoplasms false-negative results are much more likely if the site of the tumour is infratentorial or mediobasal. In patients with stroke the incidence of false-negative results depends on the stroke-scan interval.

\section{Introduction}

The value of a technique lies in a knowledge of its limitations, and this is particularly true in brain scanning, which is accepted by many as a blanket test of intracranial lesions. Certain intracranial lesions may give false-negative results, and a knowledge of the pattern is important clinically in the choice of appropriate diagnostic procedures.

In this paper a review is presented of the false-negative brain scans in a series of 847 patients with proved intracranial lesions, all of whom had undergone cerebral scintigraphic examination in the course of other investigations and treatment in the Wessex Neurological Centre, Southampton.

\section{Patients and Methods}

A survey was made of the final clinical diagnosis of about 2,000 patients subjected to brain scanning, and 847 were found in whom intracranial lesions had been proved to be present. The cerebral scintigrams of these patients were then reviewed and the results studied in relation to the size, site, and nature of the intracranial lesions. Care was taken to ensure that there was no reasonable doubt about the final diagnosis, though histological proof was not always available. For example, angiographic tumour staining followed by clinical deterioration and death was accepted evidence of a glioma or, in the presence of a

Wessex Neurological Centre, Southampton

E. H. BURROWS, M.RAD., D.M.R.D., Consultant Neuroradiologist primary cancer elsewhere, metastatic malignancy. Clinical recovery from a stroke with a positive-to-negative brain scan sequence was labelled as a cerebrovascular accident; a diagnosis of intracerebral haematoma was made only if blood was demonstrated by lumbar puncture or burr-hole biopsy.

All the patients were examined with a rectilinear scanner (Magna Scanner V, Picker) and 52 with a gamma camera (Pho-Gamma, Nuclear-Chicago) as well. The examinations were performed as carefully as possible, in order to eliminate technical causes of failure. The radiopharmaceuticals used were ${ }^{197} \mathrm{Hg}$-chlormerodrin, ${ }^{99} \mathrm{~m} \mathrm{Tc}$-pertechnetate, and ${ }^{113 \mathrm{~m} I n-}$ DTPA. In addition to radioindium scanning, 108 patients were double-scanned with one of the other substances as well. Computer aids were not used, either in obtaining or interpreting the results.

\section{Findings}

Of the 847 proved intracranial lesions, 118 (13.9\%) showed no abnormal uptake on brain scanning-that is, false-negative results. The latter were distributed as follows: 616 neoplasms, $74(12.0 \%) ; 211$ vascular lesions, $44(20.9 \%) ; 20$ inflammatory lesions, 0 . The incidence in individual lesions is given in Table $\mathrm{I}$, and their topographical distribution in Table II. These results are analysed in detail.

TABLE I-Brain Scanning in 847 Proved Intracranial Lesions: Histology of 118 False-negative Lesions

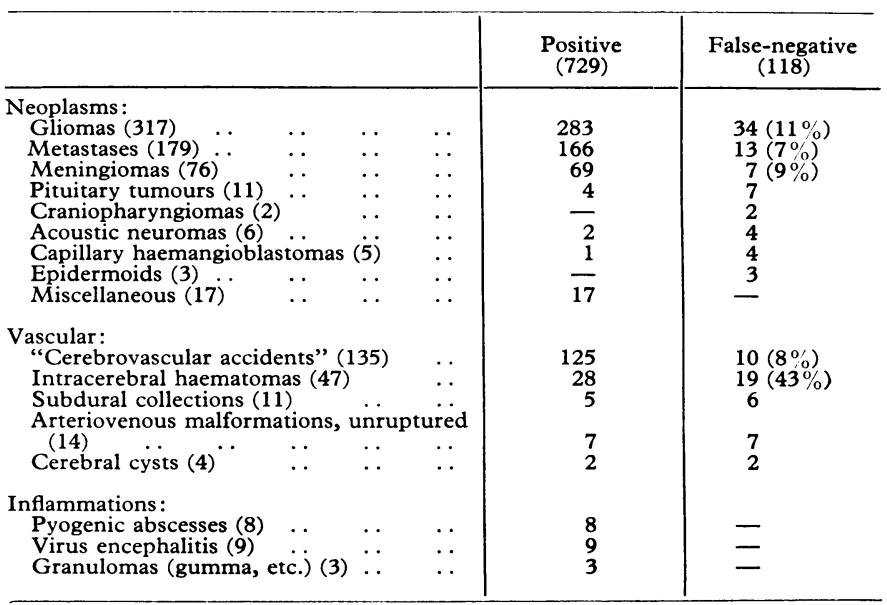


TABLE II-Brain Scanning in 847 Proved Intracranial Lesions: Topography of 118 False-negative Lesions

\begin{tabular}{|c|c|c|c|}
\hline Region & \multirow{2}{*}{$\begin{array}{c}\text { No. of Cases } \\
214 \\
224 \\
36 \\
233\end{array}$} & \multicolumn{2}{|c|}{ False-negative Cases } \\
\hline $\begin{array}{lll}\text { Frontal } & \ldots & \\
\text { Parietal } & \ldots & \\
\text { Occipital } & \ldots & \\
\text { Temporal } & \ldots & \text {. }\end{array}$ & & $\begin{array}{ll}10 & \left(4 \cdot 7^{\circ}\right) \\
11 & \left(4 \cdot 9^{\circ}{ }_{0}^{\circ}\right) \\
\frac{11}{29} & \left(12 \cdot 4^{\circ}\right)\end{array}$ & $50 / 707=7 \cdot 1 \%$ \\
\hline $\begin{array}{l}\text { Corpus callosum } \\
\text { Sella-suprasellar } \\
\text { Pineal-thalamic } \\
\text { Infratentorial }\end{array}$ & $\begin{array}{l}18 \\
20 \\
21 \\
50\end{array}$ & $\begin{aligned} 2 & (11 \%) \\
13 & (65 \%) \\
14 & (67 \%) \\
32 & (64 \%)\end{aligned}$ & $61 / 109=56 \%$ \\
\hline $\begin{array}{l}\text { Dural spaces } \\
\text { Vault and scalp }\end{array}$ & $\begin{array}{l}15 \\
16\end{array}$ & $\begin{array}{ll}6 & (40 \%) \\
1 & (0.6 \%)\end{array}$ & \\
\hline
\end{tabular}

Gliomas (34).-Analysis of the 34 false-negative gliomas showed that only three were situated in a cerebral hemisphere; the remaining 31 were on the floor of the skull (Fig. 1, hatched area). Fifteen were classified according to the Kernohan grading of malignancy: 11 were grade 1-2 and 4 grade 3-4 neoplasms. All four malignant gliomas lay in a temporal lobe.

Meningiomas (7).-All seven tumours were on the floor of the skull, one in the suprasellar region (Fig. 2), five on a lesser sphenoid wing, and one in a cerebellopontine angle. All except one were histologically benign. The infratentorial meningioma measured almost $4 \mathrm{~cm}$ in diameter and the others between 1.4 and $3 \mathrm{~cm}$. Two sphenoid wing meningiomas that gave false-

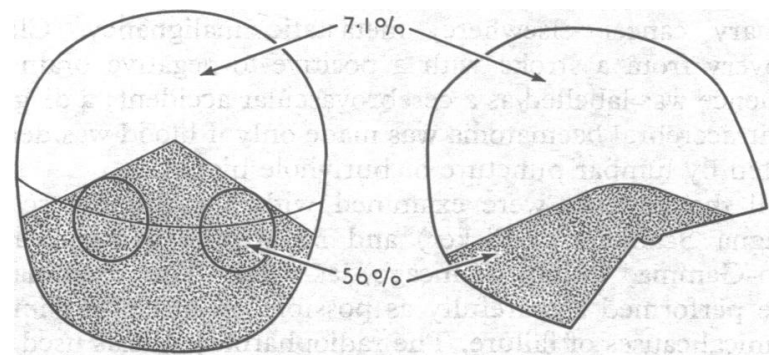

FIG. 1-Topography of false-negative brain scans. Shaded area represents the midbrain and cerebellum, also parts of the corpus callosum and temporal lobes. See Table II for derivation of percentages. negative results with radiomercury-197 were subsequently shown with radioindium (Fig. 3).

Metastases (13).-All except one were infratentorial in situation, mostly in the vermis of the cerebellum. It is likely that the false-negative result in the supratentorial deposit, a parietal lobe metastasis, was caused by poor technique.

Pituitary and Suprasellar Tumours (13).-All were midline neoplasms: chromophobe adenoma (7), craniopharyngioma (2), third-ventricle glioma (2), meningioma (1), and colloid cyst of third ventricle (1). The dimensions of all could be accurately measured on pneumoencephalography (Fig. 4): five exceeded $5 \mathrm{~cm}$ in diameter and the remainder were about $2 \mathrm{~cm}$, including three wholly intrasellar expanding tumours; the colloid cyst measured $1 \mathrm{~cm}$ in diameter.

Acoustic Neurofibromas (4).- - One of these tumours measured $3 \mathrm{~cm}$ in diameter; the other three were under $2 \mathrm{~cm}$. All six acoustic neurofibromas in this series were examined with radiomercury-197.

Capillary haemangioblastomas (4).-All four of these infratentorial tumours exceeded $2 \mathrm{~cm}$ in size, one was $5 \mathrm{~cm}$ in diameter. The fifth case in the series, which showed extensive abnormal uptake, occupied virtually the entire posterior cranial fossa.

Epidermoids (2).-Both tumours were infratentorial; the one in a cerebellopontine angle was not significantly space-occupying, but the fourth-ventricle cholesteatoma measured $6 \mathrm{~cm}$ in diameter (Fig. 2). A third cholesteatoma, involving the diploë of the skull and classified under vault and scalp lesions, also gave a false-negative result.

"Cerebrovascular Accident" (10).-Because of the need to obtain objective proof of the diagnosis, the false-negative cases in this group represent only those patients in whom carotid angiography revealed a cause of the stroke-namely, occlusion of the internal carotid or middle cerebral artery. Of the 10 cases three were examined within six days of the stroke and four over 60 days after it (see Discussion).

Intracerebral Haematoma (19). - In addition to proof of intracranial blood, the mass itself was identified in most patients by cerebral angiography, pnuemography, or craniotomy. Twelve haematomas were frontotemporal and three parietal in situation

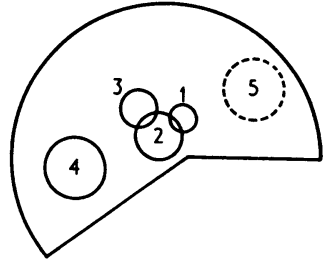

FIG. 2-Size of false-negative brain scans. (1) Meningioma in midline $(2 \mathrm{~cm})$. (2) Pituitary chromophobe adenoma $(5 \mathrm{~cm})$ (3) Malignant astrocytoma of thalamus $(4 \mathrm{~cm})$. (4) Cholesteatoma of fourth ventricle $(6 \mathrm{~cm})$ (5) Calcified astrocytoma of frontal lobe (about $5 \mathrm{~cm}$ ).
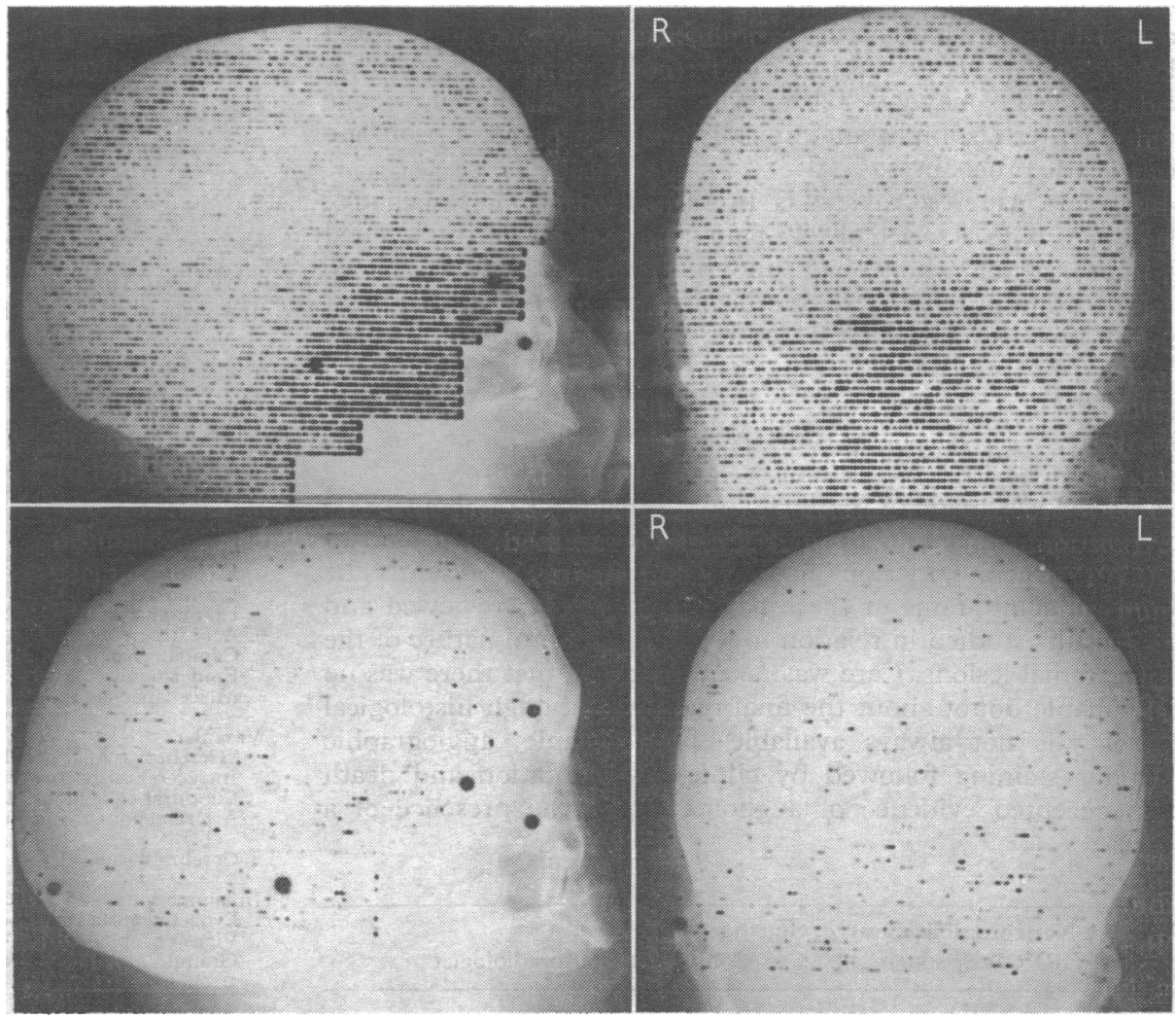
and the remaining four lay in the depths of a cerebral hemisphere. Of the 19 cases six were examined within five days of the haemorrhage and four more than 60 days after it.

Subdural Collections (6).- There were two large subdural hygromas deforming the skull and four subdural haematomas of varying stages of chronicity. In one of the latter the falsenegative result was probably due to poor technique; in the other three patients the haematomas were respectively $0.5,1.2$, and $2.5 \mathrm{~cm}$ thick, as measured on carotid angiography.

Arteriovenous Malformation, Unruptured (7).- The seven cases were distributed as follows: frontal, one; deep midline, three; and infratentorial, three. Only one was smaller than $2 \mathrm{~cm}$ in area. In the infratentorial cases the lesions seemed to occupy most of the posterior cranial fossa.

Cerebral Cysts (2).-Both these unusual, apparently nonneoplastic lesions were situated in the parietal lobe, and both measured over $3 \mathrm{~cm}$ in diameter. Fig. 5 illustrates a third case of parietal "cyst," not included in the present series, which also gave false-negative results. At operation a nodule of gliomatous tissue was found-astrocytoma grade 2-3.

Vault and Scalp Lesions (1).-This case was a cholesteatoma exhibiting typical radiological appearances and involving a 7 by $4-\mathrm{cm}$ area of the skull vault. Other skull lesions that gave positive uptake were Paget's disease, fibrous dysplasia of bone, osteomyelitis, giant osteoma of the frontal sinus (? infected), and lytic neoplastic metastases.

\section{Discussion}

Several factors contribute to false-negative results in cerebral scintigraphy, notably: (1) technical factors such as the use of inferior scintigraphic equipment, poor technique, and inappropriate radiopharmaceutical; (2) biological factors such as the size, site, and nature of the pathological process; and (3) the timing of the examination, particularly in non-neoplastic lesions.

\section{TECHNICAL FACTORS}

Even the most careful attention to all aspects of scintigraphic technique will not eliminate false-negative results entirely; it will only help to reduce them. On the other hand, it is easy to produce a false-negative result through carelessness or the use of unsuitable or inappropriate materials. Continual technical advances have led to a situation in which the choice of the particular counting method used (gamma camera or rectilinear scanner) is relatively unimportant, provided only that the particular apparatus is utilized to full advantage (Burrows, 1972). Similarly, careful comparative studies (Burrows, 1971) have shown that no particular radionuclide is strikingly superior to any other, though the short-lived substances, and particularly radiotechnetium, possess considerable practical advantages. Analysis of the results suggests that they are slightly more accurate than radiomercury-197, particularly in the detection of basal meningiomas (Fig. 3). However, it must be emphasized that the resulting increase in diagnostic accuracy is only marginal. The impression was gained, from the results in the 108 patients examined with two radioisotopes and in the smaller series examined with both gamma camera and the rectilinear scanner, that no variation of any existing technical criterion is likely to diminish significantly the proportion of false-negative results.

A careful technique of examination probably contributes more
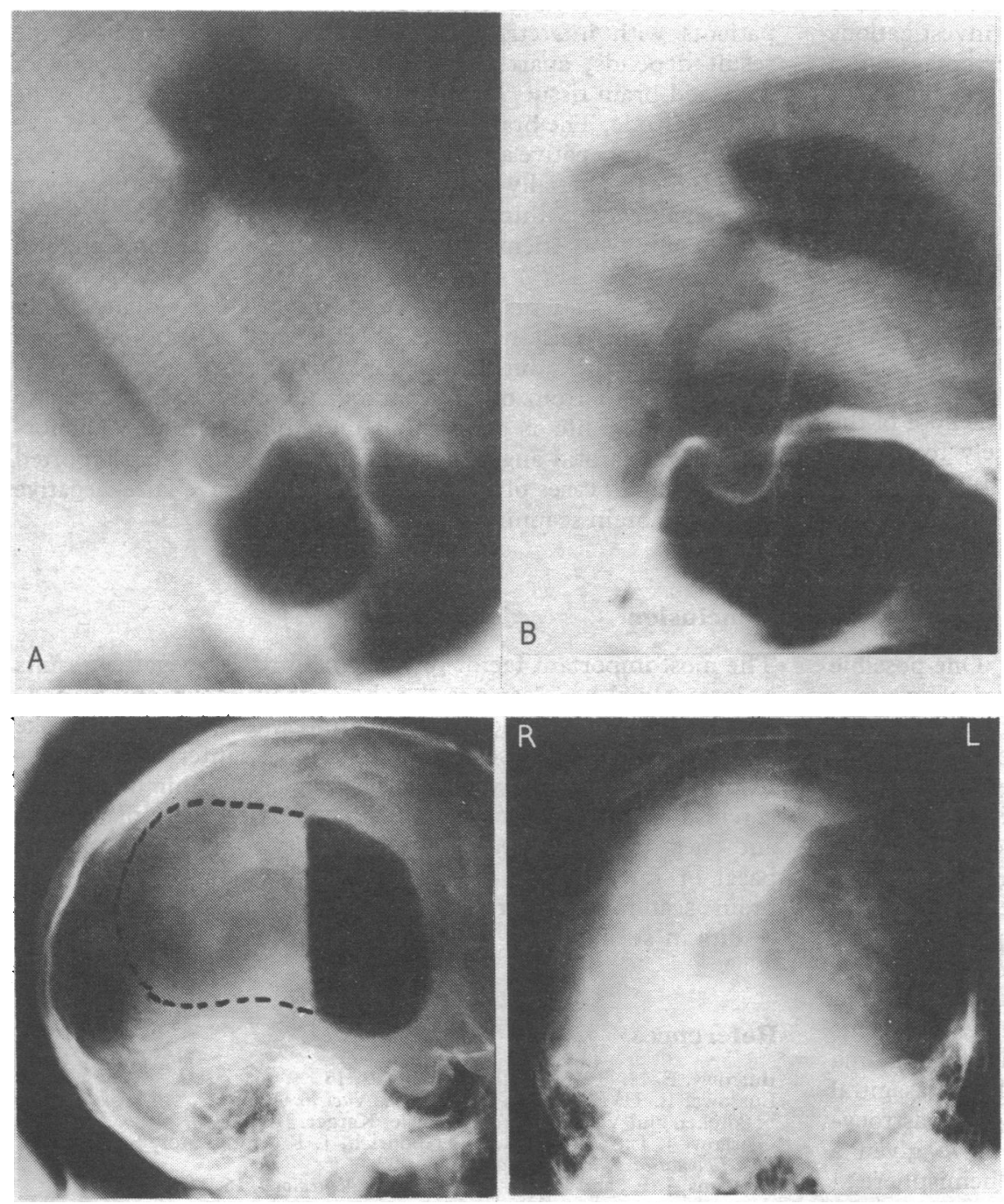

FIG 5-Gliomatous cyst replacing the posterior half of left cerebral hemisphere. Brain scan negative. Skull radiographs: pituitary fossa shows evidence of raised intracranial pressure. 


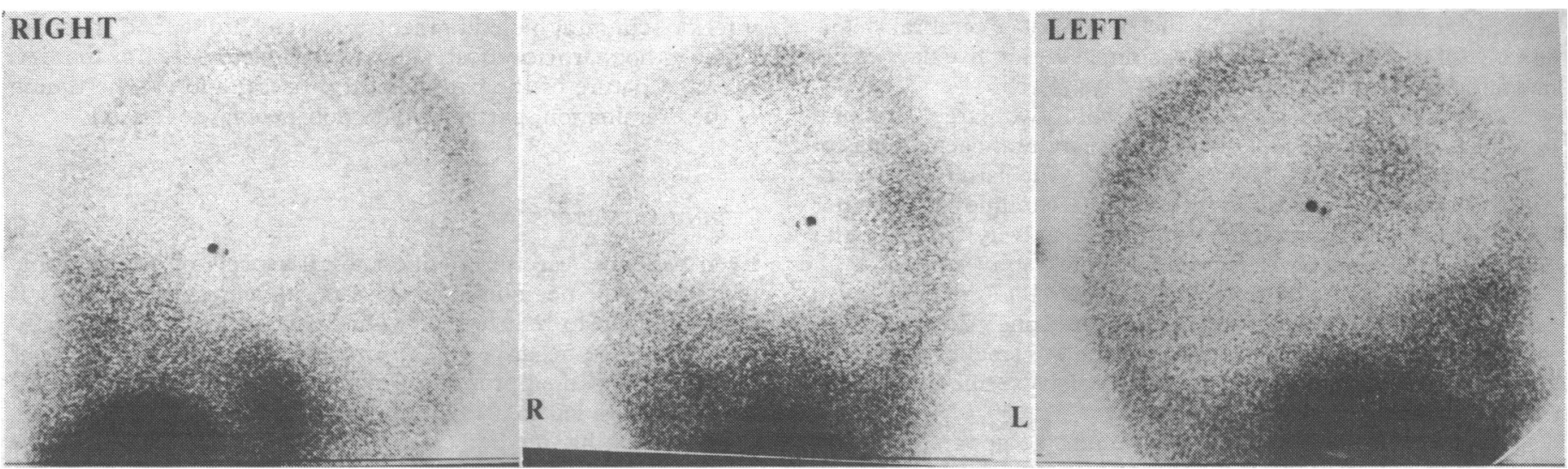

FIG. 6-Ependymoma of the left frontal convexity, well localized in the left lateral and anterior scintiphotos (right and mide
right lateral scintiphoto (left), illustrating lack of "shine-through" of gamma camera. (Black spot off-centre is an artefact.)

than any other factor to ensuring that this proportion is low. The following points merit attention: sedation of the patient, particularly infants and smaller children, to ensure non-movement of the head during the examination; anatomical positioning of the head for individual views; and a speed of traverse and a spacing of the linear pathway, in rectilinear scanning, appropriate to the radioisotope used. Failure to position the head correctly may lead to abnormal foci being missed. The gamma camera, in particular, lacks the qualities that ensure tumour "shine-through" (Fig. 6).

No brain scan should ever be interpreted without previous viewing of adequate skull and chest radiographs of recent date. Firm adherence to this rule, while unlikely to influence observer judgement to any great extent in the interpretation of the brain scan, ensures that a certain proportion of false-negative results will be detected immediately and further investigations recommended. This approach therefore represents sound clinical sense and it is a powerful argument for placing brain scanning in the charge of the neuroradiologist.

\section{BIOLOGICAL FACTORS}

The most important factor influencing the result of the examination is the nature of the lesion itself-its size, its situation, and its histology. On these variables, rather than on the radioisotope or apparatus used, will the accuracy of the result depend.

Site.-This is the overriding consideration. Analysis of the results of this series shows clearly that a lesion on the floor of the skull, including the posterior cranial fossa, is likely to go undetected, irrespective of its size or nature (Fig. 1, Table II). Nearly all the lesions in the suprasellar and pineal regions and the posterior cranial fossa listed in Table II were neoplasms of a type and size which, had they been situated in or over a cerebral hemisphere, would almost certainly have given positive results. The failure to detect them may therefore be unrelated to the biological characteristics of the lesions themselves. One possible technical limitation may be the increased physiological accumulation of radioisotope in the soft tissues overlying the skull floor, and in a few cases the use of a larger dose of a short-lived radioisotope permitted the lesion to be detected through it (Fig. 3).

Size.-Given optimal conditions of collimation, no lesion smaller than 1 in $(2.5 \mathrm{~cm})$ in diameter is likely to be found by the methods of detection at present available. However, considerably larger lesions may give false-negative results owing to their situation and histological nature. Fig. 2 illustrates five such lesions: in four of these the extent of the growth was outlined by pneumography, and in the fifth, a frontal astrocytoma, a resected surgical specimen was available. It seems likely that in Case 1 a $2-\mathrm{cm}$ diameter suprasellar meningioma would have been detected had it been lying in the parasagittal gutter. Similarly in Case 3 a 4-cm diameter malignant astrocytoma of the thalamus would almost certainly have given a positive result if it had been situated in a cerebral hemisphere.
Histology.-Fig. 4 contrasts two tumours of about equal size and situation, one an optic-pathway glioma and the other a suprasellar meningioma. The glioma gave a false-negative result but the meningioma a positive one. Since both patients were examined with the same gamma camera and identical doses of radioisotope, the only point of difference was their histological composition. However, a study of Tables I and II forces the conclusion that histological differences between neoplasms are less important than their situation within the skull for obtaining accurate scintigraphic results.

Timing of Examination ("Stroke-Scan Interval").-In nonneoplastic lesions, particularly those of a vascular naturenamely, infarcts and intracerebral haematomas - the timing of the examination is an additional factor which appreciably raises the proportion of false-negative results. Serial brain scanning of patients with infarction or haematomas has shown that the result depends, apart from the site and size of the area of damaged brain tissue, on the interval that has elapsed since the initial episode. The brain scan made immediately after the stroke is likely to be negative and then to become positive within a week, and if repeated serially it shows a gradual decline in radioactivity to normal levels within six to eight weeks (Williams and Beiler, 1966). Of the 47 patients with proved intracranial haematomas in this series, $19(43 \%)$ gave false-negative results. In the larger series of 135 patients with "cerebrovascular accidents" only $10(8 \%)$ were false-negative, a somewhat misleading figure occasioned by the difficulty in proving the presence of an infarction. Apart from brain scanning, the only way of doing so objectively in life is to demonstrate the presence of abnormalities on carotid angiography. Glasgow et al. (1965) reported a series of 129 cases of stroke in which $55 \%$ gave false-negative results on brain scanning.

\section{Conclusion}

The most important factor responsible for false-negative results in intracranial neoplasms is the site of the tumour, and in nonneoplastic vascular lesions the time interval between the stroke and the investigation (so-called "stroke-Scan interval"). A negative brain scan probably excludes a neoplasm within or over the cerebral hemispheres (especially if combined with plain skull and chest radiographs), but the reliability of the test in mediobasal or infratentorial neoplasms is only about $50 \%$. Serial brain scanning will reduce the incidence of false-negative results in stroke cases.

\section{References}

Burrows, E. H. (1971). Neuroradiology, 2, 15.

Burrows, E. H. (1972). In Progress in Nuclear Medicine, vol. 1, ed. E. J. Potchen and V. R. McCready. Basle, Karger. In press. Glasgow, J. L., Currier, R. D., Goodrich, J. K., and Tutor, F. T. (1965). fournal of Nuclear Medicine, 6, 902.

Williams, J. L., and Beiler, D. D. (1966). Neurology, 16, 1139. 